\title{
Withanolide E sensitizes renal carcinoma cells to TRAIL-induced apoptosis by increasing cFLIP degradation
}

\author{
CJ Henrich ${ }^{\star, 1,2}$, AD Brooks ${ }^{2,3}$, KL Erickson ${ }^{1,4}$, CL Thomas $^{1}$, HR Bokesch $^{1,2}$, P Tewary ${ }^{2,3}$, CR Thompson ${ }^{3}$, RJ Pompei ${ }^{3}$, KR Gustafson ${ }^{1}$, \\ JB McMahon ${ }^{1}$ and TJ Sayers ${ }^{2,3}$
}

Withanolide E, a steroidal lactone from Physalis peruviana, was found to be highly active for sensitizing renal carcinoma cells and a number of other human cancer cells to tumor necrosis factor-related apoptosis-inducing ligand (TRAIL)-mediated apoptosis. Withanolide E, the most potent and least toxic of five TRAIL-sensitizing withanolides identified, enhanced death receptor-mediated apoptotic signaling by a rapid decline in the levels of CFLIP proteins. Other mechanisms by which TRAIL sensitizers have been reported to work: generation of reactive oxygen species (ROS), changes in pro-and antiapoptotic protein expression, death receptor upregulation, activation of intrinsic (mitochondrial) apoptotic pathways, ER stress, and proteasomal inhibition proved to be irrelevant to withanolide $E$ activity. Loss of CFLIP proteins was not due to changes in expression, but rather destabilization and/or aggregation, suggesting impairment of chaperone proteins leading to degradation. Indeed, withanolide $E$ treatment altered the stability of a number of HSP90 client proteins, but with greater apparent specificity than the well-known HSP90 inhibitor geldanamycin. As cFLIP has been reported to be an HSP90 client, this provides a potentially novel mechanism for sensitizing cells to TRAIL. Sensitization of human renal carcinoma cells to TRAIL-induced apoptosis by withanolide E and its lack of toxicity were confirmed in animal studies. Owing to its novel activity, withanolide $E$ is a promising reagent for the analysis of mechanisms of TRAIL resistance, for understanding HSP90 function, and for further therapeutic development. In marked contrast to bortezomib, among the best currently available TRAIL sensitizers, withanolide E's more specific mechanism of action suggests minimal toxic side effects. Cell Death and Disease (2015) 6, e1666; doi:10.1038/cddis.2015.38; published online 26 February 2015

The use of tumor necrosis factor-related apoptosis-inducing ligand (TRAIL) in cancer therapy has long been an attractive goal given its reported ability to induce apoptosis in cancer cells but not normal cells in a variety of oncologic malignancies. ${ }^{1-4}$ Unfortunately, TRAIL has recently been reported to have other, non-apoptotic signaling properties, ${ }^{5}$ and the development of TRAIL resistance is common in many types of cancer. ${ }^{1-4,6}$ Bortezomib and other proteasome inhibitors may be the best agents currently available for reversing resistance to TRAIL, ${ }^{1,4,7,8}$ but bortezomib has long been known to be quite toxic., ${ }^{1,4,7-9}$ Thus, the search for compounds able to sensitize otherwise resistant cancer cells to TRAIL-induced apoptosis has accelerated in recent years. ${ }^{1-4,6,10}$ High-throughput screening of natural product libraries ${ }^{11}$ followed by isolation and characterization of active components has yielded a number of natural products able to sensitize resistant cancer cells to TRAIL. ${ }^{12,13}$ A series of TRAIL-sensitizing withanolides from the indigenous South American plant Physalis peruviana have now been identified, including four not previously identified as TRAIL sensitizers. Withanolides comprise a family of $\mathrm{C}_{28}$ plant steroids many of which induce apoptosis in several tumor cell lines. ${ }^{14,15}$
A number of mechanisms of action identified for TRAIL sensitizers, ${ }^{1,6,8,10,16,17}$ including generation of reactive oxygen species (ROS), induction of ER stress, inhibition of heat shock proteins, proteasomal inhibition and more general effects on transcription, translation, and signal transduction, have also been reported as possible mechanisms for the antitumor cell activities of withanolides. ${ }^{18-27}$ Withanolide $E$ (the most active and abundant TRAIL-sensitizing withanolide isolated in this work) synergizes with TRAIL to induce cancer cell apoptosis in vitro and in vivo via enhanced death receptor-mediated TRAIL signaling. Its mechanism of action, cFLIP degradation, appears to be mediated by specific modulation of HSP90 function.

\section{Results}

Withanolides are TRAIL-sensitizing constituents of an active natural product extract. The most abundant and highest potency withanolide purified and characterized from the $P$. peruviana extract was withanolide $E$. The structures and activities of this and several other withanolides as TRAIL sensitizers are shown in Figure 1. Withanolide A, withanone,

\footnotetext{
${ }^{1}$ Molecular Targets Laboratory, NCl-Frederick, Frederick, MD, USA; ${ }^{2}$ Basic Research Program, Leidos Biomedical Research, Inc., Frederick National Laboratory for Cancer Research, Frederick, MD, USA; ${ }^{3}$ Laboratory for Experimental Immunology and Cancer Inflammation Program, NCl-Frederick, Frederick, MD, USA and ${ }^{4}$ Department of Chemistry, Clark University, Worcester, MA, USA

*Corresponding author: CJ Henrich, Basic Research Program, Leidos Biomedical Research, Inc., Frederick National Laboratory for Cancer Research, Building 538, Room 167, NCl-Frederick, Frederick 21702, MD, USA. Tel: +1 301846 6054; Fax: +1 301846 6919; E-mail: henrichcj@mail.nih.gov

Abbreviations: DISC, death-inducing signaling complex; CFLIP, cellular FLICE-like inhibitory protein; NAC, N-acetyl cysteine; ROS, reactive oxygen species; TRAIL, tumor necrosis factor-related apoptosis-inducing ligand; WE, withanolide $E$; WA, withanolide A; WFA, withaferin $A$

Received 10.10.14; revised 09.1.15; accepted 13.1.15; Edited by G Giaccone
} 
withaperuvin, and 12-deoxywithastramonolide were inactive (up to $40 \mu \mathrm{M}$ ) with or without TRAIL. Withanolide $\mathrm{E}$ had the least growth inhibitory effect as a single agent $(5.7 \%$ reduction in cell number at $10 \mu \mathrm{M}$ as compared with $10.9 \%$ for $4 \beta$-hydroxy withanolide E, $21.9 \%$ for withanolide S, 39.5\% for dehydrowithanolide $E$, and $44.0 \%$ for withaferin A).

The effects of withanolide $E$ on growth of renal carcinoma cells are shown in Figure 2. Figure 2 a clearly demonstrates that withanolide E eliminates long-term survival of ACHN cells, but only in the presence of TRAIL, thus confirming that the combination leads to cell death rather than simply growth inhibition. The increased apparent potency of withanolide $\mathrm{E}$ +TRAIL in longer-term experiments suggests that these cells are irreversibly committed to cell death (i.e., they do not subsequently recover upon removal of withanolide $E$ and TRAIL) even though they may be detectable by 2,3-Bis(2methoxy-4-nitro-5-sulfophenyl)-5-[(phenylamino)carbonyl]2H-tetrazolium hydroxide (XTT), MTS, or ATP assays after shorter duration (1 day) treatment. Withanolide $\mathrm{E}$ was also a very effective TRAIL sensitizer for two other renal carcinoma lines (Figure $2 b$ ), but only modestly affected untransformed human renal epithelial (HRE) cells, even at very high TRAIL concentrations (Figure 2c). Similarly, withanolide E alone had minimal effects on the growth of 22 other diverse cancer cell lines, but several TRAIL-resistant cancer cell lines became TRAIL-sensitive after withanolide $E$ treatment (see Supplementary Information). Withanolide $E$ also had only minor effects on morphology of ACHN and CAKI cells (see Supplementary Information).

Withanolide E enhances normal death receptor signaling. To assess whether sensitization of ACHN cells to TRAIL by withanolide $\mathrm{E}$ occurs via activation of the extrinsic (i.e., TRAIL-dependent) apoptosis pathway, several components of 'normal' TRAIL signaling were measured (Figure 3). Withanolide E sensitized ACHN cells to apoptosis induced by agonistic antibodies to death receptors 4 and 5; thus, indicating that it affected death receptor activation (Figures $3 a$ and b). Sensitization of ACHN cells to TRAIL-induced apoptosis required caspase activation (blocked by ZVADFMK, a caspase inhibitor - Figure 3c). Withanolide E enhanced the TRAIL-dependent sequential activation of caspase 8 ( $>15$-fold increase after $4 \mathrm{~h}$ followed by $4 \mathrm{~h}$ TRAIL - Figure 3d, confirmed by immunoblot - Figure $3 \mathrm{~g}$ ) and caspase 3 (>10-fold increase after $24 \mathrm{~h}$ - Figure $3 e$ ), followed by DNA fragmentation (Figure 3f), indicative of end-stage apoptosis. Withanolide $E$ alone had minor effects on each of these measurements.

However, withanolide $\mathrm{E}$ alone significantly reduced the levels of cellular cFLIP (Figure $3 \mathrm{~g}$ ). TRAIL-dependent death-
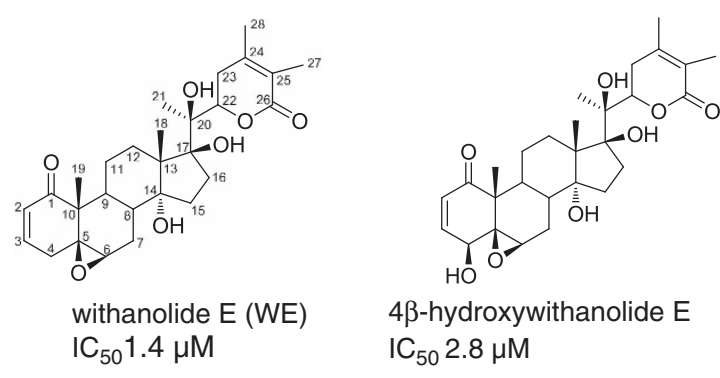

$4 \beta$-hydroxywithanolide $\mathrm{E}$ $\mathrm{IC}_{50} 2.8 \mu \mathrm{M}$

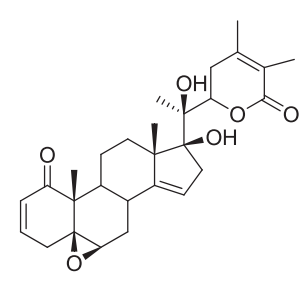

dehydrowithanolide $\mathrm{E}$ (tentative structure) $\mathrm{IC}_{50} 4.5 \mu \mathrm{M}$
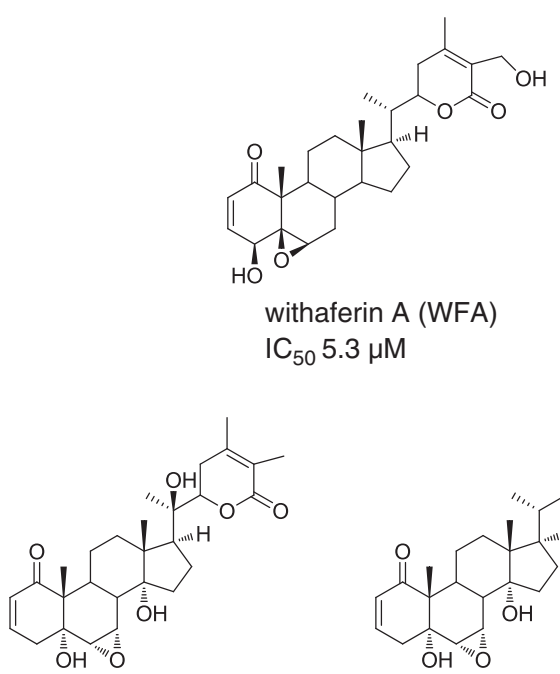

withanolide A (WA)

$\mathrm{IC}_{50}>40 \mu \mathrm{M}$

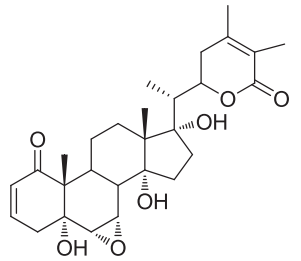

withanone

$\mathrm{IC}_{50}>40 \mu \mathrm{M}$

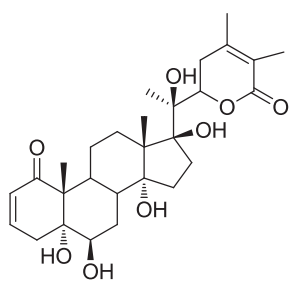

withanolide S $\mathrm{IC}_{50} 12.1 \mu \mathrm{M}$

Figure 1 Withanolides vary in their ability to sensitize ACHN cells to TRAIL. IC 50 values (average, $n=2-6$ ) were calculated from dose-response data and indicate $50 \%$ reduction in cell number by withanolide+TRAIL compared with untreated cells 
a

Withanolide $\mathrm{E}(\mathrm{nM})$
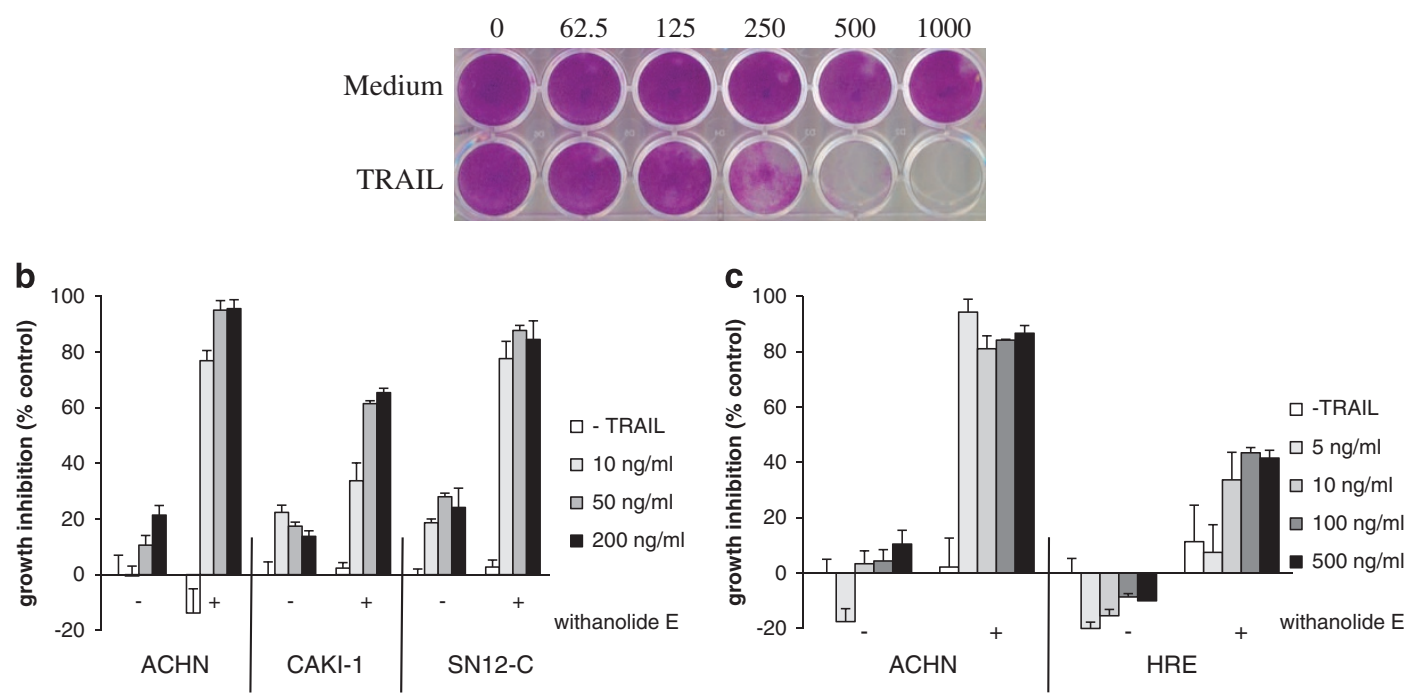

Figure 2 Effects of withanolide E on renal carcinoma cells. (a) ACHN cells were treated $4 \mathrm{~h}$ with withanolide $\mathrm{E}$, then TRAlL overnight followed by washing and medium replacement. After 5 days, cells were fixed, stained, and photographed. (b and $\mathbf{c}$ ) Renal carcinoma (ACHN, CAKl-1, SN12-C) or human renal epithelial (HRE) cells in 96-well plates were treated for $4 \mathrm{~h}$ with $1 \mu \mathrm{M}$ withanolide $\mathrm{E}$ followed by $24 \mathrm{~h}$ with the indicated concentrations of TRAIL and relative cell numbers assessed. Error bars represent S.D. $(n=3)$

inducing signaling complex (DISC) formation was robust in the presence of withanolide $\mathrm{E}$, albeit with decreased CFLIP and led to enhanced caspase 8 recruitment, resulting in an increase in the caspase 8:cFLIP ratio (Figure 3h). The control for this experiment, bortezomib, induced greater TRAILdependent caspase 8 recruitment than did withanolide $\mathrm{E}$, albeit with greater DISC-associated cFLIP as well. The significantly reduced level of cFLIP in the DISC following withanolide $\mathrm{E}$ treatment would suggest increased DISCassociated caspase 8 activation, leading to amplification of TRAIL-induced apoptotic signaling. Together these results confirm that treatment with withanolide E+TRAIL rapidly and irreversibly commits $\mathrm{ACHN}$ cells to apoptosis via a rapid drop in the total cellular levels of cFLIP and amplification of the apoptotic signaling from the DISC.

Effects of withanolide $E$ on mechanisms of TRAIL sensitization. Sensitization of TRAIL-resistant cells to TRAIL-induced apoptosis can occur via a variety of mechanisms and effects, ${ }^{6-10,28}$ including changes in the levels of pro- and antiapoptotic proteins; ER stress; activation of the intrinsic (mitochondrial) pathway; generation of ROS; death receptor upregulation; proteasome inhibition; DISC dysregulation; caspase activation; cFLIP downregulation. In marked contrast to bortezomib, expression of a panel of proteins previously reported to be important for intrinsic apoptosis signaling and TRAIL-driven apoptosis was not significantly affected by withanolide $\mathrm{E}$ alone (Figure $4 \mathrm{a}$ and Supplementary Information), and indicators of ER stress (CHOP and GRP78) were unaffected by withanolide $E$ (Figure 4b). Similarly, withanolide $E$ does not induce mitochondrial depolarization except in the presence of TRAIL (i.e., late-stage apoptosis - Figure 4c). By contrast, doxorubicin was previously identified as a TRAIL sensitizer in the initial screen, ${ }^{11}$ and it activates the intrinsic mitochondrial pathway $^{29}$ as demonstrated by induction of mitochondrial depolarization (data not shown), thus confirming that the ACHN cells utilized here can be sensitized to TRAIL, at least in part, via induction of the intrinsic apoptotic pathway. Both withanolide $E$ and the inactive withanolide $A$ induced transient increases in ROS, peaking at $4 \mathrm{~h}$, but by $24 \mathrm{~h}$ there was no longer a measurable ROS increase (Figure 4d). However, pretreatment with withanolide $\mathrm{E}$ for $24 \mathrm{~h}$ (i.e., after ROS decayed) followed by short incubation with TRAIL resulted in complete cell killing (data not shown). The presence of TRAIL had no effect on withanolide-induced ROS generation (data not shown). NAC treatment eliminated the cell killing effect of withanolide $E$ in the presence of TRAIL, but two other antioxidants (vitamin C and Trolox) had no such effect (Figure 4e). Consistent with previous reports of reactivity between withanolides and NAC, ${ }^{30} \mathrm{NAC}$ formed a covalent chemical adduct with withanolide $\mathrm{E}$ (see Supplementary Information). Thus, ROS generation is unlikely to contribute to the TRAIL-sensitizing activity of withanolide $\mathrm{E}$ and chemical reactivity rather than ROS quenching explains NAC's inhibitory activity. Death receptor (DR4 and DR5) expression was only modestly affected by withanolide $\mathrm{E}$ (Figure 4f), in contrast to previously reported induction of increased DR4 and DR5 expression by the TRAIL sensitizer bortezomib. ${ }^{31}$ Withanolide $\mathrm{E}$ treatment did not lead to inhibition of proteasomal activity or accumulation of ubiquitinated proteins (see Supplementary Information) nor did it affect the levels of caspases (Figure 3).

Withanolide E induces cFLIP degradation. Of the TRAILsensitization mechanisms assessed, only withanolide E-induced cFLIP downregulation was observed, specifically rapid reduction in total cellular cFLIP (Figure $3 g$ ) without affecting caspase 8 or FADD (Figures $3 g$ and $4 a$ ). TRAILdependent caspase activation was not required for cFLIP downregulation, but residual $\mathrm{CFLIP}_{\mathrm{L}}$ was completely eliminated on subsequent addition of TRAIL (Figure $3 \mathrm{~g}$ ). 

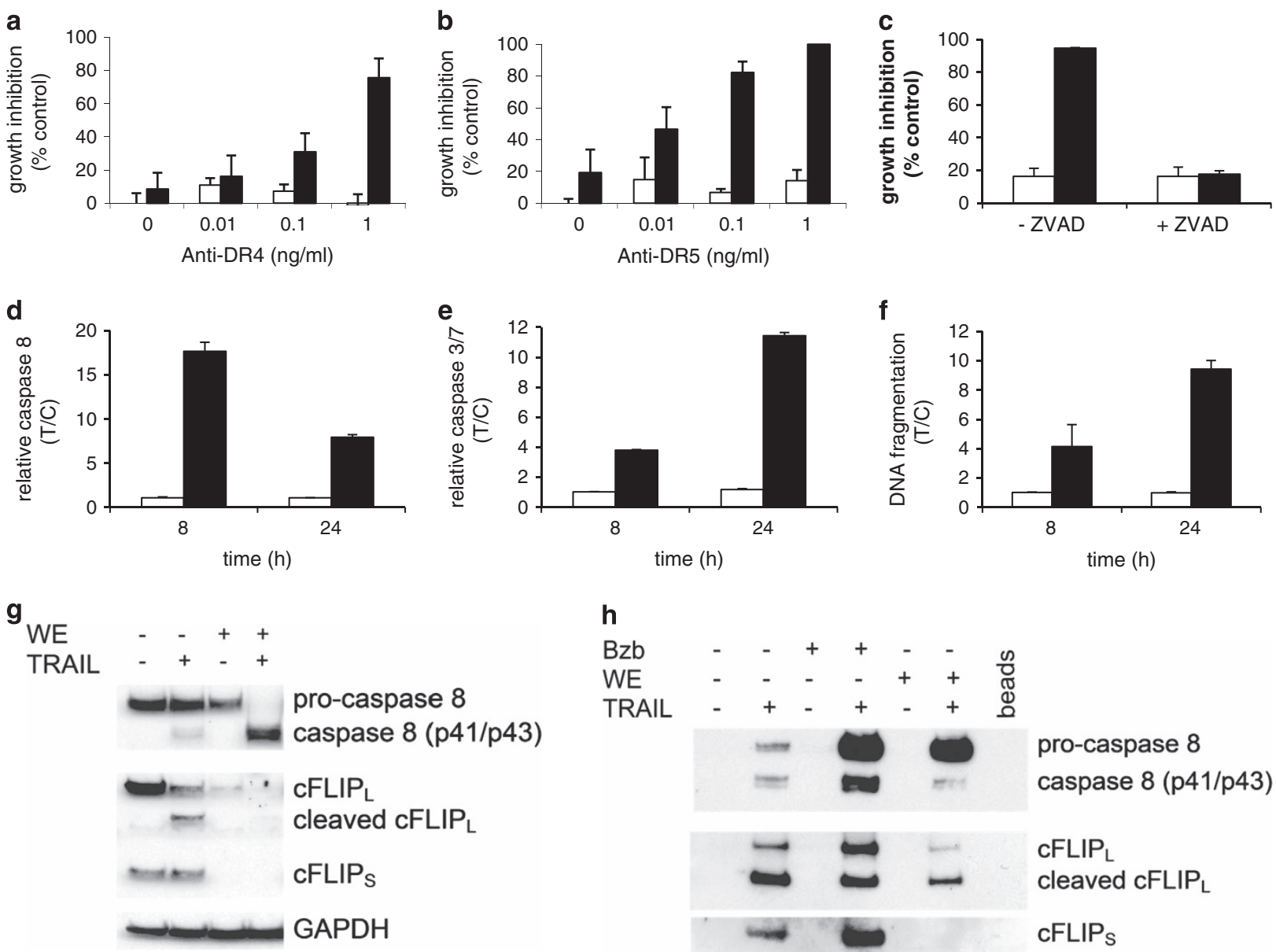

Figure 3 Withanolide E enhances TRAIL-induced extrinsic apoptotic pathway in ACHN cells. Cells were treated without (open bars) or with (filled bars) withanolide $\mathrm{E}$ for $3-4 \mathrm{~h}$ followed by agonistic anti-DR4 (a) or DR5 (b) antibodies for 20-24 h. For panels c-f, cells were treated $4 \mathrm{~h} \pm$ withanolide $\mathrm{E}$ (WE) then $4 \mathrm{~h}$ (for a total of $8 \mathrm{~h}$ ) or $24 \mathrm{~h} \pm \mathrm{TRAlL}$ in the continued presence of WE. Open bars: withanolide $\mathrm{E}$ only; black: withanolide $\mathrm{E}$ followed by TRAIL. (c) Cells were pretreated with ZVAD-FMK to block caspase activation followed by WE, then TRAIL. Caspase 8 (d) and caspase 3 (e) activities, and DNA fragmentation (f) were assessed using commercial kits. (g) Caspase cleavage and cFLIP degradation were assessed by immunoblot (total treatment time, $8 \mathrm{~h}$ ). (h) TRAIL-dependent DISC formation was assessed in withanolide E- or bortezomib (Bzb - control with known activity)treated ACHN cells (overnight treatment followed by biotinylated TRAIL at $4{ }^{\circ} \mathrm{C}$, cell lysis, DISC precipitation and immunoblot). Error bars represent S.D. $(n=3$ )

Significant reduction of cFLIP was seen as early as $4 \mathrm{~h}$ after treatment with as low as $1 \mu \mathrm{M}$ withanolide $\mathrm{E}$ and by $24 \mathrm{~h}$, the short form was undetectable and the long form barely detectable (Figure 5a). Downregulation of cFLIP protein can occur rapidly upon blocking cFLIP mRNA transcription. ${ }^{32-36}$ However, withanolide $E$ treatment did not have a significant effect on cFLIP mRNA levels (see Supplementary Information), making it likely that the drop in CFLIP is due to increased degradation. cFLIP reduction could result from dysregulation/aggregation, first leading to altered subcellular distribution and solubility and, ultimately, degradation. When proteasomal activity was inhibited by bortezomib, withanolide E-induced degradation of cFLIP was blocked and cFLIP accumulated, but cFLIP redistributed to a Triton-insoluble cellular compartment (Figure 5b). Bortezomib alone does not have this effect. These results suggest withanolide E-induced misfolding and/or aggregation of cFLIP. This, together with the reported effects of other withanolides on heat shock proteins ${ }^{19,37}$ and reports that cFLIP is an HSP90 client protein, ${ }^{38}$ led to an investigation of the effects of withanolide $\mathrm{E}$ on HSP90 client proteins. The inactive withanolide A served as a negative control, and it elicited no changes in any of the proteins assessed (Figure 5a). HSP70 expression was increased by withanolide $E$ and by geldanamycin, (Figures $5 a$ and c), consistent with inhibition of HSP90, ${ }^{39}$ but neither compound affected levels of CDK2, a protein not chaperoned by HSP90 (Figures $5 \mathrm{a}$ and $\mathrm{c}$ ). Several other established HSP90 client proteins, RAF1, CDK4, glucocorticoid receptor, and cyclin D1, were downregulated by geldanamycin but were unaffected or only modestly affected by withanolide $E$ (Figure $5 \mathrm{c}$ ). Both withanolide $\mathrm{E}$ and geldanamycin reduced pAKT levels. By contrast, only withanolide $E$ resulted in reduced cFLIP levels (Figure 5c). Geldanamycin did not efficiently sensitize ACHN cells to TRAIL (data not shown). The withanolide E effects on PAKT, HSP70, and cFLIP all had similar time- and dose-dependencies, but expression of HSP90 itself was unaffected (Figure 5a). Inhibition of proteasomal activity by bortezomib blocked the withanolide E-induced degradation of pAKT without affecting total AKT (Figure $5 d$ ), suggesting that withanolide $E$ affects pAKT protein stability. It has been reported that HSP90 can preferentially stabilize activated forms of protein kinases 
a

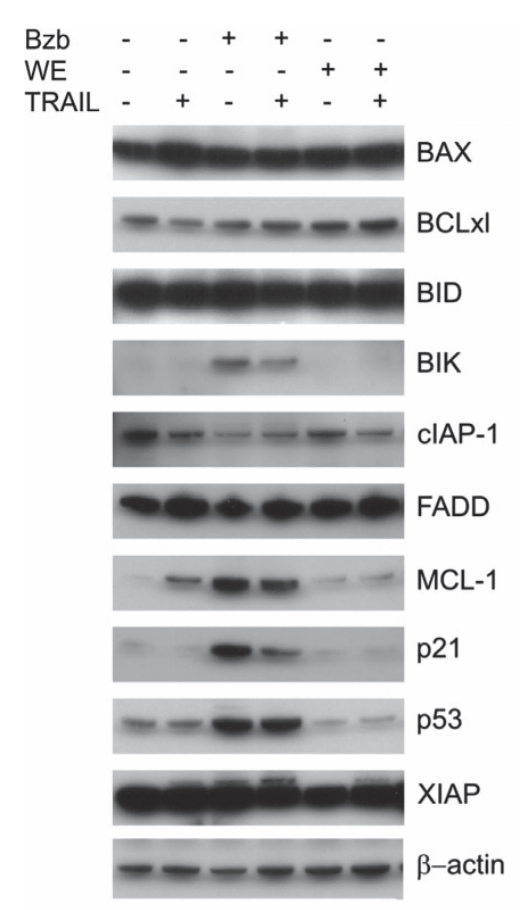

C
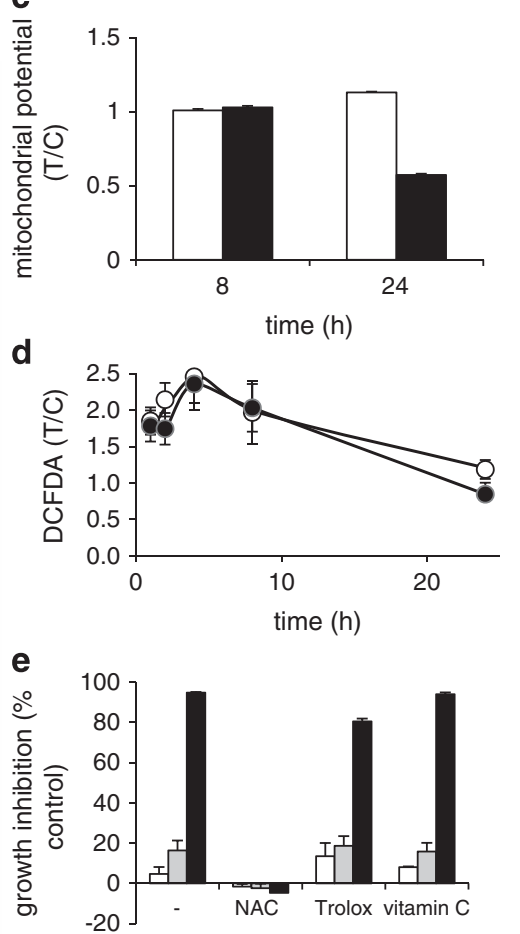

f
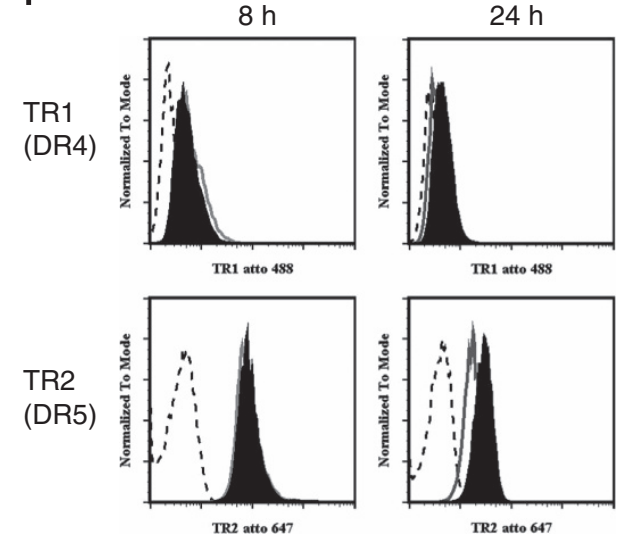

b

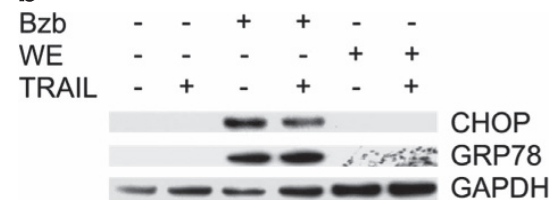

Figure 4 Withanolide $\mathrm{E}$ effects on TRAIL sensitization mechanisms. (a) Effects of withanolide $\mathrm{E}$ on expression of pro- and antiapoptotic proteins. ACHN cells were treated $24 \mathrm{~h}$ with bortezomib $(20 \mathrm{nM})$ or withanolide $\mathrm{E}$, then \pm TRAlL $(50 \mathrm{ng} / \mathrm{ml})$ and immunoblot. (b) ACHN cells were treated \pm bortezomib (Bzb, positive control) or withanolide $\mathrm{E}$ (WE) followed by \pm TRAlL (total treatment time, $8 \mathrm{~h}$ ) and immunoblot analysis of ER stress markers. (c) Cells were treated $4 \mathrm{~h} \pm$ withanolide $\mathrm{E}$ then $4 \mathrm{~h}$ (for a total of $8 \mathrm{~h}$ ) or $24 \mathrm{~h} \pm$ TRAIL in the continued presence of WE. Open bars: withanolide $\mathrm{E}$ only; black: withanolide $\mathrm{E}$ followed by TRAIL. Mitochondrial potential was assessed (JC-1 assay). (d) Cells were treated with withanolide $\mathrm{E}$ (solid symbols) or withanolide A (inactive control, open symbols) and ROS generation estimated with DCFDA. (e) ACHN cells were pretreated with NAC $(10 \mathrm{mM})$, Trolox $(200 \mu \mathrm{M})$ or vitamin C (vitC, $200 \mu \mathrm{M})$ followed by withanolide $\mathrm{E}(2 \mathrm{~h})$, then TRAIL $(24 \mathrm{~h})$. Open bars: withanolide $\mathrm{E}$ only; gray bars: TRAIL only; black bars: withanolide E+TRAIL. (f) ACHN cells were treated for $8 \mathrm{~h}$ or $24 \mathrm{~h}$ with $10 \mu \mathrm{M}$ withanolide $\mathrm{E}$ and assessed for expression of TRAIL receptors 1 and 2 by FACS (FACS Caliber, BD Biosciences). Antibodies used were (anti-TRAlL-R1 (human), mAb (HS101) (ATTO 488) or anti-TRAlL-R2 (human), mAb (HS201) (ATTO 647N) or mouse lgG1 atto488 or mouse lgG1atto 647 (Adipogen)). Dashed line represents isotype control, solid line DMSO-treated cells, and filled line withanolide E-treated cells. Error bars represent S.D. $(n=3-4)$

(for example, $\mathrm{Met}^{40}$ ). Thus, it is reasonable to conclude that the loss of pAKT as a result of withanolide $E$ treatment is HSP90-related rather than a result of changes in phosphorylation/dephosphorylation. These results also suggest that HSP90 inhibition may only generate TRAIL sensitization when it leads to cFLIP degradation. Inclusion of withaferin A, known to bind to HSP90, ${ }^{19,37}$ at a sub-sensitizing/sub-toxic concentration $(0.5 \mu \mathrm{M})$ completely eliminated the TRAIL-sensitizing activity of withanolide $E$ $(<5 \%$ growth inhibition in the presence of withanolide $E+$ withaferin $A+$ TRAIL).

In vivo enhancement of TRAIL-induced antitumor effects by withanolide E. As withanolide $E$ promoted TRAILinduced apoptosis in ACHN cells in vitro, the combination of withanolide $\mathrm{E}$ and an agonist antibody to TRAIL death receptor DR5 was assessed in vivo. Figure 6 demonstrates the in vivo efficacy of withanolide $\mathrm{E}$ as a TRAIL sensitizer in a mouse model system. Intra-tumor administration of the combination of withanolide $\mathrm{E}$ and drozitumab (DR5 agonistic antibody) was more effective in decreasing tumor progression than either agent alone. Intraperitoneal administration of the combination provided a superior therapeutic benefit over either agent alone in long-term tumor survival studies. The combination of agents resulted in $>55 \%$ of the mice having no detectable palpable tumor 150 days after the start of therapy. A further prolonged follow-up of some of these surviving mice for over 250 days showed no further signs of tumor, consistent with a complete and sustained tumor regression in these individuals. The animals were monitored for indicators of overt toxicities and were weighed twice weekly. No obvious toxicities were observed at any stage during the administration of these treatment schedules (data not shown). 
a

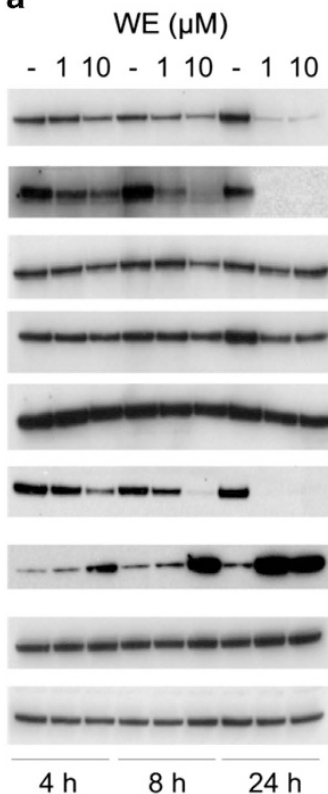

\section{b}

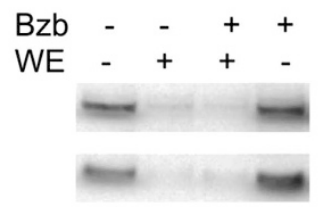

Triton extract
WA $(\mu \mathrm{M})$

$-110-110-110$
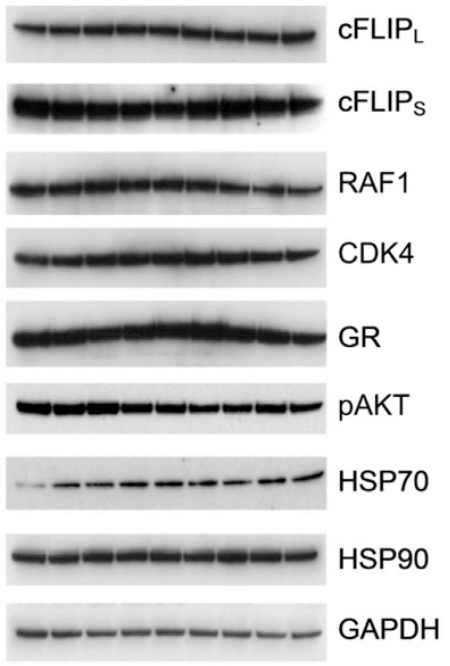

$4 \mathrm{~h} \quad 8 \mathrm{~h} \quad 24 \mathrm{~h}$
C
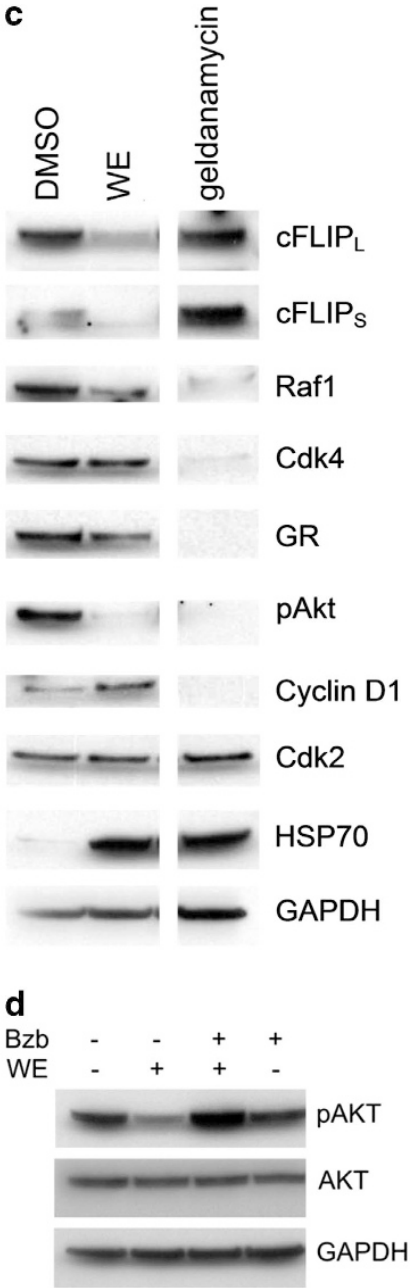

Figure 5 Withanolide E affects CFLIP and HSP90 client proteins. (a) Cells were treated for $4-24 \mathrm{~h}$ with 1 or $10 \mu \mathrm{M}$ withanolide $\mathrm{E}$ (WE) or withanolide A (WA) followed by immunoblot detection of indicated proteins. (b) Cells were treated $\pm \mathrm{WE} \pm$ bortezomib $(40 \mathrm{nM})$ and processed by extraction with a Triton X-100-containing extraction buffer or with SDS-containing sample buffer to yield 'Triton ext' and 'Total ext' samples, respectively, and analyzed for cFLIP by immunoblot. (c) Cells were treated for $8 \mathrm{~h}$ with geldanamycin (positive control) or WE followed by immunoblot. (d) ACHN cells were treated \pm WE \pm bortezomib (Bzb) followed by immunoblot analysis of pAKT and total AKT
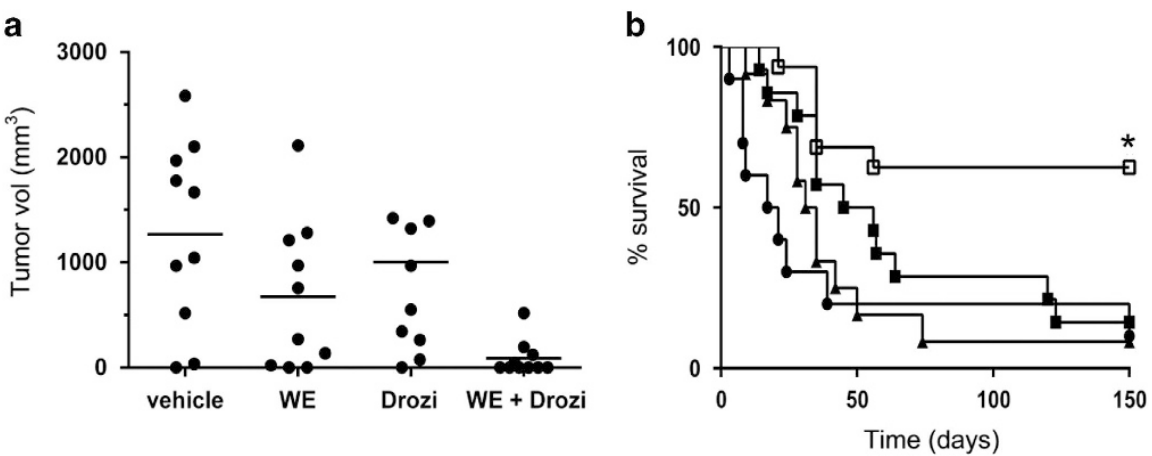

Figure 6 Withanolide $E$ enhances death receptor-induced apoptosis in vivo. Effects of withanolide $E$ and drozitumab (DR5 agonist antibody) on growth of ACHN cell-derived tumors in athymic mice was assessed as described in the text. (a) Tumor size at 75 days (intratumor), (b) survival up to 150 days (intraperitoneal) - pooled from two separate experiments with similar findings. Numbers of mice were: vehicle control $(\boldsymbol{\bullet}, n=10)$, withanolide $\mathrm{E}(\boldsymbol{\Lambda}, n=10)$, drozitumab $(\boldsymbol{\square}, n=14)$, withanolide $\mathrm{E}$ plus drozitumab $(\square, n=16) .{ }^{*} P<0.05$ 


\section{Discussion}

Withanolide $E$ was the most effective TRAIL sensitizer, but least toxic, of the withanolides assessed. Withanolide $\mathrm{E}$ enhanced the extrinsic/death receptor-dependent apoptotic pathway rather the intrinsic mitochondrial pathway and, as a single agent, had no significant pro-apoptotic effects. Its most significant effect was a rapid decrease in cellular cFLIP. cFLIP is a major regulator of TRAIL signaling, ${ }^{33,41,42}$ and loss of cFLIP can lead to increased TRAIL-induced apoptosis. ${ }^{34-36}$ Rapid loss of cFLIP protein and only minor changes in cFLIP mRNA suggest increased degradation in response to withanolide $\mathrm{E}$. Blocking degradation drove residual CFLIP into a less soluble cellular compartment consistent with denaturation or aggregation. Thus cFLIP dysregulation may prime cells for response to death receptor activation. Recent reports have demonstrated that cFLIP has a critical role in tissue homeostasis by preventing apoptosis and necroptosis. Conditional knockout of cFLIP in skin, ${ }^{43}$ intestinal epithelia, ${ }^{44}$ or liver ${ }^{45,46}$ resulted in cell death in response to endogenous death ligands. CFLIP is essential in preventing inappropriate cell death in normal tissues, suggesting that a reduction in cFLIP may increase apoptosis in neoplastic disease. Given the literature reports of other potential mechanisms of action for TRAIL sensitizers, characterization of the TRAIL-sensitizing activity of withanolide $E$ included assessment of effects on apoptotic protein expression, proteasomal activity, generation of ROS, ER and mitochondrial stress, and death receptor expression. As demonstrated in the results section and cited references, each of these mechanisms can be activated in the present experimental system but withanolide $\mathrm{E}$ does not utilize them to sensitize cells to TRAIL. ROS generation was not a factor in the TRAIL-sensitizing activity of withanolide $E$, and withanolide $E$ had little effect on the other phenomena. Interestingly, one other withanolide, withaferin $A$, has been previously identified as a TRAIL sensitizer. ${ }^{47}$ In contrast to withanolide $\mathrm{E}$, withaferin $\mathrm{A}$ did not significantly affect cFLIP. However, consistent with previous reported targets, ${ }^{48}$ withaferin $A$ had significant effects on ER stress and on cellular morphology and inhibited proteasomal activity (data not shown and Supplementary Information) and it was much more toxic to ACHN cells as a single agent than was withanolide E. Critically, siRNA-mediated reduction of cFLIP results in sensitization of renal carcinoma cells to TRAILmediated apoptosis, ${ }^{34}$ demonstrating that the reduction in cFLIP alone is sufficient to sensitize these cells. Thus, cFLIP dysregulation was the only relevant TRAIL-sensitizing activity observed for withanolide $\mathrm{E}$.

Heat shock proteins, particularly HSP90, have been reported to mediate some cellular responses to withanolides. ${ }^{18,19,37}$ Withanolides may interact with and destabilize HSP90, with a resultant elimination of client proteins via the proteasome. It has previously been reported that siRNA-mediated reduction of HSP90 sensitized glioblastoma cells to TRAIL apoptosis by effects on cFLIP, suggesting that CFLIP is an HSP90 client protein and that reduced levels of HSP90 prevented appropriate trafficking of cFLIP to the TRAIL DISC. ${ }^{38}$ Thus, the effects of withanolide $E$ are consistent with HSP90 inhibition leading to misfolding and/or aggregation of cFLIP culminating in cFLIP degradation.
Redistribution of cFLIP to less soluble (i.e., Triton-insoluble) cellular fraction(s) is consistent with this interpretation. Treatment of $\mathrm{ACHN}$ cells with withanolide $\mathrm{E}$ clearly affected the levels of some HSP90 client proteins, specifically cFLIP and phosphoAKT. Inhibitors of AKT activation did not sensitize ACHN cells to TRAIL (data not shown). Thus loss of phosphoAKT is probably a result of HSP90 inhibition by withanolide $\mathrm{E}$, but not a driver of withanolide E-induced cFLIP downregulation or TRAIL sensitization in these cells. In contrast to geldanamycin, withanolides (including withanolide E) have been reported to bind to the C-terminal domain of HSP90. ${ }^{19}$ This may account in part for the greater selectivity of withanolide $\mathrm{E}$ with regard to HSP90 inhibition and the spectrum of client proteins affected. Withaferin $A$, a known HSP90-binding compound, blocked the TRAIL-sensitizing effect of withanolide $E$. Its ability to block withanolide $E$ is consistent with the suggestion that the TRAIL-sensitizing effect of withanolide E may be HSP90-dependent and that the two compounds compete for the same or nearby binding site(s) and/or that withaferin $\mathrm{A}$ induces a conformational change such that withanolide $\mathrm{E}$ is no longer able to bind. Preliminary results suggest that withanolide E can bind to HSP90 and follow-up experiments are underway to identify the nature of this interaction as well as to investigate other mechanisms of cFLIP downregulation.

Insufficient numbers of withanolides have been assessed as TRAIL sensitizers to develop significant structure-activity relationships (SAR), but small structural differences clearly lead to significant diversity in activity. However, the cytotoxicity/apoptosis-inducing activity of withanolides has been the subject of several SAR studies. The C2,C3 double bond in ring $A$ is absolutely required and the C5,C6 epoxide moiety enhances toxicity, whereas withanolides with a $\mathrm{C} 6, \mathrm{C} 7$ epoxide are relatively innocuous. ${ }^{18-20,30,49-51}$ As shown in this work (Figure 1), withanolides with the C5,C6 epoxide were active as TRAIL sensitizers, whereas those with the C6,C7 epoxide were inactive. The $\mathrm{C} 4$ hydroxyl enhances toxicity in many cell types, ${ }^{18,19,50}$ but had little, if any, effect on sensitization of ACHN cells to TRAIL (compare withanolide $E$ and $4 \beta$-hydroxy withanolide $\mathrm{E}$ ). It has been reported that the $\mathrm{C} 17$ and $\mathrm{C} 20$ hydroxyls may slightly reduce withanolide toxicity. ${ }^{49}$ These structural features generally correlate with the toxicity of the compounds in the absence of TRAIL as seen above. SAR for cytotoxicity/apoptosis and for HSP90 effects are also correlated. ${ }^{18,19,30}$ Recent studies demonstrated that a hydroxyl at C4 affects reactivity with HSP90 and specificity for other targets. Withaferin $A$ and $4 \beta$-hydroxy withanolide $E$ were reported to disrupt the interaction of HSP90 with CDC37, but withanolide $\mathrm{E}$ (lacking the $\mathrm{C} 4$ hydroxyl) was much less effective. $^{19}$ Thus the structural features that differ between withanolide $E$ and the significantly more toxic (and less potent as a TRAIL sensitizer) withaferin A may result in differential specificity for HSP90 and client proteins, including cFLIP. Additional experiments are underway to better characterize the interactions between TRAIL-sensitizing withanolides and HSP90 proteins and may contribute to future design of low toxicity HSP90-targeted agents.

A number of human cancer cells were sensitized to TRAILinduced apoptosis by withanolide $\mathrm{E}$, whereas others remained resistant. The molecular basis for this difference is unknown 
and is being investigated but may be related to their relative expression of cFLIP. For example, cFLIP is reported to have a central role in conferring TRAIL resistance to melanoma ${ }^{52,53}$ and glioblastoma, ${ }^{54,55}$ and several TRAIL-resistant melanoma and glioblastoma cell lines were sensitized by withanolide $E$ to TRAIL-induced apoptosis (see Supplementary Information). Thus, withanolide $\mathrm{E}$ may prove to be a powerful reagent for assessing the relative importance of cFLIP levels for TRAIL resistance in cancer cells, as well as providing a means of overcoming cFLIP-associated TRAIL resistance. The effects of withanolide $\mathrm{E}$ on cFLIP protein stability rather than transcription may also allow for greater specificity and more focused therapeutic applications. The identification of withanolide $\mathrm{E}$ as a novel and selective modifier of HSP90:client protein interactions will provide a better understanding of the role of HSP90 in TRAIL signaling. The contrast between withanolide $\mathrm{E}$ and bortezomib, among the best available TRAIL sensitizers, is also significant. Bortezomib is wellknown to exhibit in vitro and in vivo toxicity, possibly because of the global effects of inhibiting the proteasome and its potency as a general cell stressor. On the other hand, withanolide $\mathrm{E}$ has minimal effects on multiple mechanisms (e.g., cell stress, mitochondrial effects, ROS, etc.) that could lead to significant toxicity and/or other side effects. Its lack of toxicity as a single agent in vitro and in vivo as well as its limited ability to induce apoptosis in normal cells at modest TRAIL concentrations bode well for its potential therapeutic utility.

Thus withanolide $\mathrm{E}$ may prove to be a powerful reagent for increasing understanding of HSP90 function as well as mechanisms of cellular resistance to TRAIL-induced apoptosis, and may have future therapeutic application in combination with the targeting of death receptor signaling in cancer cells.

\section{Materials and Methods \\ Chemicals and reagents. Withanolides were purified from $P$. peruviana extracts ( $\mathrm{NCl}$ Natural Products Repository) as described in Supplementary Data and/or obtained from the $\mathrm{NCl}$ Developmental Therapeutics Program and/or from Chromadex (Irvine, CA, USA). Sources of other reagents were XTT (NSC 601519) from the NCl Drug Synthesis and Chemistry Branch; bortezomib (NIH Pharmacy, Bethesda, MD, USA); recombinant TRAIL ligand (168 amino acid TNF homologous extracellular domain - Peprotech, Rocky Hill, NJ, USA); Z-VAD-FMK (BioMol, Plymouth Meeting, PA, USA); cell culture media and additives (Cellgro (Manasses, VA, USA), Hyclone (Logan, UT, USA), Sigma (St. Louis, MO, USA), or Invitrogen (Carlsbad, CA, USA)); BCA protein assay kits (Pierce/Thermo, Rockford, IL, USA); other reagents from Sigma. Chemical structures were drawn using ChemDraw (CambridgeSoft Corp., Cambridge, MA, USA) using structural information from the PubChem database (http://pubchem.ncbi.nlm.nih.gov/).}

Cell growth assays. ACHN, CAKI-1, and SN12-C cell lines (NCI) and HRE cells were from (Lifeline Cell Technology, Frederick, MD, USA), and were maintained as recommended by source institutions. Growth was assayed as described. ${ }^{11}$ In brief, cells were allowed to attach overnight ( 3500 cells/well, 384-well or 5000 cells/ well, 96-well plates) followed by $2-4 \mathrm{~h}$ with compounds or DMSO. TRAIL was added and cell numbers were estimated $(24 \mathrm{~h})$ using XTT ${ }^{11}$ or MTS (Promega, Madison, WI, USA). For analysis of ROS involvement, N-acetyl cysteine (NAC, $10 \mathrm{mM}$ ), Trolox $(200 \mu \mathrm{M})$, or vitamin $\mathrm{C}(200 \mu \mathrm{M})$ was added just before compounds and remained in the plates throughout. NAC interfered with XTT and MTS (data not shown), so cell numbers were estimated using a luminescence ATP assay (Promega). For caspase inhibition, cells were pretreated with $100 \mu \mathrm{M}$ ZVAD-FMK. To assess death receptor utilization, agonistic anti-DR4 (Alexis/Enzo, Farmingdale, NY, USA) or DR5 (R\&D Systems, Minneapolis, MN, USA) replaced TRAIL.

Unless otherwise noted, in all experiments, withanolide and TRAIL concentrations were $1 \mu \mathrm{M}$ and $20-40 \mathrm{ng} / \mathrm{ml}$, respectively.
Long-term survival of ACHN renal cancer cells. Clonal outgrowth experiments were performed to confirm cell death rather than just growth inhibition. ${ }^{11}$ Withanolide $\mathrm{E}$ was added for $3 \mathrm{~h}$, followed by TRAlL $(500 \mathrm{ng} / \mathrm{ml})$. The next day, plates were washed and medium replaced. After 5 days, plates were methanol fixed, stained with Crystal Violet, and visualized.

Immunoblot. ACHN cells $\left(2 \times 10^{6}\right.$ cells/well, 6 -well plates) were extracted for SDS-PAGE and immunoblot analysis as described. ${ }^{7}$ In some experiments, cells were lysed with either a Triton X-100-containing extraction buffer or with SDScontaining RIPA buffer (both containing $40 \mu \mathrm{M}$ ZVAD). Triton supernatants and total cell extracts were subjected to SDS-PAGE and immunoblot. Primary antibodies, detection reagents, and methods are listed in Supplementary Information.

Apoptotic signaling. ACHN cells (3500 cells/well, 384-well plates) were treated for $4 \mathrm{~h}$ followed by TRAIL (4-24h). Assessment of caspase 8 or 3 utilized Caspase-Glo 8 or $3 / 7$ assay kits (Promega). DNA fragmentation was estimated using the Roche Cell Death ELISA system. Relative mitochondrial potential was assessed using the ratiometric fluorescent dye JC-1. ${ }^{56}$ Cells (6-well plates, 500000 cells/well) were treated $4 \mathrm{~h}$ followed by TRAIL. After $30 \mathrm{~min}$ at $37^{\circ} \mathrm{C}$ with $2 \mu \mathrm{M} \mathrm{JC}-1$ (added immediately after dilution), green and red fluorescence data acquired on an $\mathrm{BD} /$ Accuri $\mathrm{C} 6$ flow cytometer and analyzed using the instrument's CFLOW software (BD/ Accuri Cytometers, San Jose, CA, USA). The fluorescence ratio is a measure of mitochondrial potential. ${ }^{56}$

ROS detection. Generation of ROS was quantitated using DCFDA, a compound that fluoresces upon oxidation by ROS. After cell treatment, $\mathrm{CM}-\mathrm{H}_{2} \mathrm{DCFDA}$ (Invitrogen) was added $(25 \mu \mathrm{M})$ for the last $1 \mathrm{~h}$ of incubation. After washing, cell-associated fluorescence was measured on a Tecan fluorometer at Ex $495 \mathrm{~nm}, \mathrm{Em} 529 \mathrm{~nm}$ (bottom read mode).

Immunoprecipitation of DISC. Cells $\left(2 \times 10^{6}\right.$ cells/well, 6 -well plates) were treated with $20 \mathrm{nM}$ Bortezomib or $1 \mu \mathrm{M}$ withanolide E overnight $\left(37^{\circ} \mathrm{C}\right)$ then $2 \mathrm{~h}\left(4^{\circ} \mathrm{C}\right)$ with $500 \mathrm{ng} / \mathrm{ml}$ biotinylated TRAlL (rhTRAlL (Peprotech) biotinylated using PlatinumLink Labeling Kit, Kreatech Diagnostics (Amsterdam, The Netherlands)). After cold PBS wash, lysis in IP DISC lysis buffer ( $30 \mathrm{mM}$ Tris-HCL pH 7.5, $150 \mathrm{mM}$ $\mathrm{NaCl}, 10 \%$ glycerol, $1 \%$ Triton X-100+protease inhibitors and $20 \mu \mathrm{M}$ Z-VAD-FMK), and equalization for protein concentration, extracts were incubated with Dynabeads MyOne Strepavidin T1 resin (Invitrogen) $\left(15 \mathrm{~min}, 4^{\circ} \mathrm{C}\right)$, washed in IP DISC lysis buffer, eluted in $2 \times$ NuPAGE LDS Sample Buffer and NuPAGE Reducing Agent (Invitrogen), and subjected to immunoblot analysis.

Animal studies. Athymic BALB/C mice were obtained from the Animal Production program (Charles River Laboratories, Inc., Frederick, MD, USA). For the generation of subcutaneous tumors, mice were injected subcutaneously with $1 \times 10^{6}$ ACHN cells that had been adapted for in vivo passage in a volume of $100 \mu$ l. After tumor volumes reached $\sim 100 \mathrm{~mm}^{3}$ ( 14 days post injection) therapy was initiated either direct injection of withanolide $\mathrm{E}$ and Drozitumab (agonist antibody to TRAIL death receptor DR5, kindly provided by Dr. Avi Ashkenazi, Genentech, South San Francisco, CA, USA ${ }^{57,58}$ ) into the tumor, or intraperitoneal injection of both agents. For intratumor injections, withanolide $\mathrm{E}$ was administered in $\mathrm{PBS} / 50 \% \mathrm{DMSO}$ in a volume of $50 \mu \mathrm{l}(20 \mathrm{mg} / \mathrm{kg})$, whereas for intraperitoneal injections, withanolide E was in DMSO in a volume of $20 \mu \mathrm{l}$ to give a dose of $20 \mathrm{mg} /$ $\mathrm{kg}$. Drozitumab in PBS was injected in a volume of $50 \mu \mathrm{l}$ for both intratumor and intraperitoneal injections to give a final dose of $5 \mathrm{mg} / \mathrm{kg}$. Control groups were treated with the vehicles alone. The weekly treatment schedule was injection of withanolide E followed the next day with drozitumab, then 1 day pause, followed by a second dose of withanolide $E$ with a second administration of drozitumab 1 day later followed by a 2 days pause. Four weekly cycles of the drugs were given. Mice were continuously monitored for any signs of distress. Two perpendicular diameters of the tumor were measured twice per week using calipers, and the tumor volume was calculated using the formula: $V=0.5 a \times b^{2}$ with $a$ and $b$ being the long and short diameters of the tumor, respectively. For survival studies, when the tumors reached greater than $500 \mathrm{~mm}^{3}$ or if the tumor had been necrotic for more than 4 weeks with no evidence of regression, mice were painlessly euthanized with $\mathrm{CO}_{2}$ as outlined in the Frederick National Laboratory for Cancer Research Animal Care and Use Committee (ACUC) guidelines.

Data presentation and analysis. Results were normalized to control (DMSO)-treated cells. Unless otherwise noted, values reported represent average 
\pm S.D. $I C_{50}$ values were estimated from dose-response curves using SigmaPlot 4-parameter logistic nonlinear regression analysis (SPSS, Inc., Chicago, IL, USA). For survival studies Kaplan-Meier plots were generated and $P$-values were obtained with the log-rank Mantel-Cox test (Prism software version 6.0 (GraphPad Software, Inc., LaJolla, CA, USA)).

\section{Conflict of Interest}

The authors declare no conflict of interest.

Acknowledgements. This project has been funded in whole or in part with Federal funds from the Frederick. Laboratory for Cancer Research, National Institutes of Health, under contract HHSN261200800001E. The content of this publication does not necessarily reflect the views or policies of the Department of Health and Human Services, nor does mention of trade names, commercial products, or organizations imply endorsement by the US Government. This research was supported (in part) by the Intramural Research Program of $\mathrm{NIH}$, Frederick National Lab, Center for Cancer Research. NCl-Frederick is accredited by AAALAC International and follows the Public Health Service Policy for the Care and Use of Laboratory Animals. Animal care was provided in accordance with the procedures outlined in the 'Guide for Care and Use of Laboratory Animals' (National Research Council; 1996; National Academy Press; Washington, DC). We thank Dr. Avi Ashkenazi (Genentech) for advice on the administration of drozitumab for in vivo studies.

1. Sayers TJ. Targeting the extrinsic apoptosis signaling pathway for cancer therapy. Cance Immunol Immunother 2011; 60: 1173-1180.

2. Martinez-Lostao L, Marzo I, Anel A, Naval J. Targeting the Apo2L/TRAIL system for the therapy of autoimmune diseases and cancer. Biochem Pharmacol 2012; 83: 1475-1483.

3. Micheau O, Shirley S, Dufour F. Death receptors as targets in cancer. Br J Pharmacol 2013; 169: $1723-1744$

4. Fulda S. Tumor-necrosis-factor-related apoptosis-inducing ligand (TRAIL). Adv Exp Med Biol 2014; 818: 167-180.

5. Azijli K, Weyhenmeyer B, Peters GJ, deJong S, Kruyt FAE. Non-canonical kinase signaling by the death ligand TRAIL in cancer cells: discord in the death receptor family. Cell Death Differ 2013; 20: 858-868

6. Dimberg LY, Anderson CK, Camidge R, Behbakht K, Thorburn A, Ford HL. On the TRAIL to successful cancer therapy? Predicting and counteracting resistance against TRAIL-based therapeutics. Oncogene 2013; 32: 1341-1350.

7. De Wilt LHAM, Kroon J, Jansen G, de Jong S, Peters GJ, Kruyt FAE. Bortezomib and TRAlL: a perfect match for apoptotic elimination of tumour cells? Crit Rev Oncol Hematol 2013; 85 363-372.

8. Lemke J, von Karstedt S, Zinngrebe J, Walczak H. Getting TRAlL back on track for cancer therapy. Cell Death Differ 2014; 21: 1350-1364.

9. Orlowski RZ, Stinchcombe TE, Mitchell BS, Shea TC, Baldwin AS, Stahl S et al. Phase I tria of the proteasome inhibitor PS-341 in patients with refractory hematologic malignancies. $J$ Clin Oncol 2002; 20: 4420-4427.

10. Hellwig CT, Rehm M. TRAIL signaling and synergy mechanisms used in TRAIL-based combination therapies. Mol Cancer Ther 2012; 11: 3-13.

11. Booth NL, Sayers TJ, Brooks AD, Thomas CL, Jacobsen K, Goncharova El et al. A cell-based high-throughput screen to identify synergistic TRAlL sensitizers. Cancer Immunol Immunother 2009; 58: 1229-1244.

12. Whitson EL, Thomas CL, Henrich CJ, Sayers TJ, McMahon JB, McKee TC. Clerodane diterpenes from Casearia arguta that act as synergistic TRAlL sensitizers. J Nat Prod 2010; 73: 2013-2018.

13. Whitson EL, Sun H, Thomas CL, Henrich CJ, Sayers TJ, McMahon JB et al. Synergistic TRAIL sensitizers from Barleria alluaudii and Diospyros maritime. J Nat Prod 2012; 75: 394-399.

14. Mirjalili MH, Moyano E, Bonfill M, Cusido RM, Palazon J. Steroidal lactones form Withania somnifera, an ancient plant for novel medicine. Molecules 2009; 14: 2373-2393.

15. Chen L-X, He H, Qiu F. Natural withanolides: an overview. Nat Prod Rev 2011; 28: 705-740.

16. Siegelin MD. Utilization of the cellular stress response to sensitize cancer cells to TRAILmediated apoptosis. Expert Opin Cell Targets 2012; 16: 801-917.

17. Menke C, Bin L, Thorburn J, Behbakht K, Ford HL, Thorburn A. Distinct TRAIL resistance mechanisms can be overcome by proteasome inhibition but not generally by synergizing agents. Cancer Res 2011; 71: 1883-1892.

18. Wang H-C, Tsai Y-L, Wu YC, Chang FR, Liu M-H, Chen WY, Wu C-C. Withanolides-induced breast cancer cell death is correlated with their ability to inhibit heat protein 90. PLoS One 2012; 7: e37764.

19. Gu M, Yu Y, Gunaherath GM, Gunatilaka AA, Li D, Sun D. Structure-activity relationship (SAR) of withanolides to inhibit Hsp90 for its activity in pancreatic cancer cells. Invest New Drugs 2014; 32: 68-74.

20. Antony ML, Lee J, Hahm ER, Kim SH, Marcus Al, Kumari V et al. Growth arrest by the antitumor steroidal lactone withaferin $A$ in human breast cancer cells is associated with downregulation and covalent binding at cysteine-303 of $\beta$-tubulin. J Biol Chem 2014; 289: 1852-1865.
21. Lee J, Hahm ER, Marcus Al, Singh SV. Withaferin A inhibits experimental epithelialmesenchymal transition in MCF-10A cells and suppresses vimentin protein level in vivo in breast tumors. Mol Carcinog 2013; doi:10.1002/mc.22110.

22. Ichikawa H, Takada Y, Shishodia S, Jayaprakasam B, Nair MG, Aggarawal BB. Withanolides potentiate apoptosis, inhibit invasion, and abolish osteoclastogenesis through suppression of nuclear factor-kappaB (NFKB) activation and $\mathrm{NF} \kappa \mathrm{B}$-regulated gene expression. Mol Cancer Ther 2006; 5: 1434-1445.

23. Mondal S, Mandal C, Sangwan R, Chandra S, Mandal C. Withanolide D induces apoptosis in leukemia by targeting the activation of neutral sphingomyelinase-ceramide cascade mediated by synergistic activation of c-Jun $\mathrm{N}$-terminal kinase and p38 mitogen-activated protein kinase. Mol Cancer 2010; 9: 239.

24. Malik F, Kumar A, Bhushan S, Khan S, Bhatia A, Suri KA et al. Reactive oxygen species generation and mitochondrial dysfunction in apoptotic cell death of human myeloid leukemia $\mathrm{HL}-60$ cells by a dietary compound withaferin $\mathrm{A}$ with concomitant protection by $\mathrm{N}$-acetyl cysteine. Apoptosis 2007; 12: 2115-2133.

25. Mayola E, Gallerne C, Esposti DD, Martel C, Pervaiz S, Larue L et al. Withaferin A induces apoptosis in human melanoma cells through generation of reactive oxygen species and down-regulation of Bcl-2. Apoptosis 2011; 16: 1014-1027.

26. Khan S, Rommeloo AW, Keikkila JJ. Withaferin A induces proteasome inhibition, endoplasmic reticulum stress, the heat shock response and acquisition of thermotolerance. PLoS One 2012; 7: e50547.

27. Khedgikar V, Kushwaha P, Gautam J, Verma A, Changkija B, Kumar A et al. Withaferin A: a proteasomal inhibitor promotes healing after injury and exerts anabolic effect on osteoporotic bone. Cell Death Dis 2013; 22: e778.

28. Ozoren N, El-Deiry WS. Defining characteristics of types I and II apoptotic cells in response to TRAIL. Neoplasia 2002; 4: 551-557.

29. Sarosiek KA, Ni Chonghaile T, Letai $\mathrm{A}$. Mitochondria: gatekeepers of response to chemotherapy. Trends Cell Biol 2013; 23: 612-619.

30. Wijeratne EM, Xu YM, Scherz-Shouval R, Marron MT, Rocha DD, Liu MX et al. Structureactivity relationships for withanolides as inducers of the cellular heat-shock response. $J \mathrm{Med}$ Chem 2014; 57 : 2851-2863.

31. Brooks D, Jocobsen KM, Li W, Shanker A, Sayers TJ. Bortezomib sensitizes human renal cell carcinomas to TRAIL apoptosis through increased activation of caspase-8 in the deathinducing signaling complex. Mol Cancer Res 2010; 8: 729-738.

32. Martin-Perez R, Niwa M, Lopez-Rivas A. ER stress sensitizes cells to TRAlL through downregulation of FLIP and Mcl-1 and PERK-dependent up-regulation of TRAIL-R2. Apoptosis 2012; 17: 349-363.

33. Okano H, Shiraki K, Inoue H, Kawakita T, Yamanaka T, Deguchi M et al. Cellular FLICE/ caspase-8-inhibitory protein as a principal regulator of cell death and survival in human hepatocellular carcinoma. Lab Invest 2003; 83: 1033-1043.

34. Brooks AD, Sayers TJ. Reduction of the antiapoptotic protein cFLIP enhances the susceptibility of human renal cancer cells to TRAIL apoptosis. Cancer Immunol Immunother 2005; 54: 499-505

35. Sharp DA, Lawrence DA, Ashkenazi A. Selective knockdown of the long variant of cellular FLICE inhibitory protein augments death receptor-mediated caspase-8 activation and apoptosis. J Biol Chem 2005; 280: 19401-19409.

36. Sayers TJ, Brooks AD, Koh CY, Ma W, Seki N, Raziuddin A et al. The proteasome inhibitor PS-341 sensitizes neoplastic cells to TRAIL-mediated apoptosis by reducing levels of c-FLIP. Blood 2003; 102: 303-310.

37. Yu Y, Hamza A, Gu M, Zou P, Newman B, Li Y et al. Withaferin A targets heat shock protein 90 in pancreatic cancer cells. Biochem Pharmacol 2010; 79: 542-551.

38. Panner A, Murray JC, Berger MS, Pieper RO. Heat shock protein $90 \alpha$ recruits FLIPs to the death-inducing signaling complex and contributes to TRAIL resistance in human glioma. Cancer Res 2007; 67: 9482-9489.

39. Trepel J, Mollapour M, Giaccone G, Neckers L. Targeting the dynamic HSP90 complex in cancer. Nat Rev Cancer 2010; 10: 537-549.

40. Miyajima N, Tsutsumi S, Sourbier C, Beebe K, Mollapour M, Rivas C et al. The HSP90 inhibitor ganetespib synergizes with the MET kinase inhibitor crizotinib in both crizotinibsensitive and -resistant MET-driven tumor models. Cancer Res 2013; 73: 7022-7033.

41. Safa AR. C-FLIP, a master anti-apoptotic regulator. Exp Oncol 2012; 34: 176-184.

42. Ozturk S, Schleich K, Lavrik IN. Cellular FLICE-like inhibitory proteins (c-FLIPs): fine tuners of life and death decisions. Exp Cell Res 2012; 318: 1324-1331.

43. Panayotova-Dimitrova D, Feoktistova M, Ploesser M, Kellert B, Hupe M, Horn S et al. cFLIP regulates skin homeostasis and protects against TNF-induced keratinocyte apoptosis. Cell Rep 2013; 5: 397-408.

44. Wittkopf N, Gunther C, Martini E, He G, Amann K, He YW et al. Cellular FLICE-like inhibitory protein secures intestinal epithelial cell survival and immune homeostasis by regulating caspase-8. Gastroenterology 2013; 145: 1369-1379.

45. Schattenberg JM, Zimmermann T, Worns M, Sprinzl MF, Kreft A, Kohl T et al. Ablation of c-FLIP in hepatocytes enhances death-receptor mediated apoptosis and toxic liver injury in vivo. J Hepatology 2011; 55: 1272-1280.

46. Piao X, Komazawa-Sakon S, Nishina T, Koike M, Piao JH, Ehlken $\mathrm{H}$ et al. c-FLIP maintains tissue homeostasis by preventing apoptosis and programmed necrosis. Sci Signal 2012; 5 : ra93.

47. Lee TJ, Um HJ, Min DS, Park JW, Choi KS, Kwon TK. Withaferin A sensitizes TRAILinduced apoptosis through reactive oxygen species-mediated up-regulation of death receptor 5 and down-regulation of c-FLIP. Free Radic Biol Med 2009; 46: 1639-1649. 
48. Vanden Berghe W, Sabbe L, Kaileh M, Haegeman G, Heyninck K. Molecular insight in the multifunctional activities of withaferin A. Biochem Pharmacol 2012; 84: 1282-1291.

49. Damu AG, Kuo PC, Su CR, Kuo TH, Chen TH, Bastow KF et al. Isolation, structures, and structure-cytotoxic activity relationships of withanolides and physalins from Physalis angulate. J Nat Prod 2007; 70: 1146-1152.

50. Zhang H, Samadi AK, Cohen MS, Timmermann BN. Anti-proliferative withanolides from Solanaceae: a structure-activity study. Pure Appl Chem 2012; 84: 1353-1367.

51. Llanos GG, Araujo LM, Jimenez IA, Moujir LM, Bazzocchi IL. Withaferin A-related steroids from Withania aristata exhibit potent antiproliferative activity by inducing apoptosis in human tumor cells. Eur J Med Chem 2012; 54: 499-511.

52. Tian F, Lu JJ, Wang L, Li L, Yang J, Li Y et al. Expression of cFLIP in malignant melanoma, and its relationship with the clincopathological features of the disease. Clin Exp Dermatol 2012; 37: 259-265.

53. Geserick P, Drewniok C, Hupe M, Haas TL, Diessenbacher P, Sprick MR et al. Suppression of cFLIP is sufficient to sensitize human melanoma cells to TRAIL- and CD95L-mediated apoptosis. Oncogene 2008; 27: 3211-3220.

54. Koschny R, Holland H, Sykora J, Haas TL, Sprick MR, Ganten TM et al. Bortezomib sensitizes primary human astrocytoma cells of WHO grades I to IV for Tumor Necrosis Factor-Related Apoptosis-Inducing Ligand-induced apoptosis. Clin Cancer Res 2007; 13: 3403-3412.

55. Bangert A, Cristofanon S, Eckhardt I, Abhari BA, Kolodziej S, Hacker S et al. Histone deascetylase inhibitors sensitize glioblastoma cells to TRAlL-induced apoptosis by c-mycmediated downregulation of cFLIP. Oncogene 2012; 31: 4677-4688.
56. Smiley ST, Reers M, Mottola-Hartshorn C, Lin M, Chen A, Smith TW et al. Intracellular heterogeneity in mitochondrial membrane potentials revealed by a J-aggregate-forming lipophilic cation JC-1. Proc Natl Acad Sci USA 1991; 88: 3671-3675.

57. Wilson NS, Yang B, Yang A, Loeser S, Marsters S, Lawrence D et al. An Fc $\gamma$ receptordependent mechanism drives antibody-mediated target-receptor signaling in cancer cells. Cancer Cell 2011; 19: 101-113.

58. Adams C, Totpal K, Lawrence D, Marsters S, Pitti R, Yee S et al. Structural and functional analysis of the interaction between the agonistic monoclonal antibody Apomab and the proapoptotic receptor DR5. Cell Death Differ 2008; 15: 751-761.

(i) Cell Death and Disease is an open-access journal published by Nature Publishing Group. This work is licensed under a Creative Commons Attribution 4.0 International License. The images or other third party material in this article are included in the article's Creative Commons license, unless indicated otherwise in the credit line; if the material is not included under the Creative Commons license, users will need to obtain permission from the license holder to reproduce the material. To view a copy of this license, visit http://creativecommons.org/licenses/by/4.0/

Supplementary Information accompanies this paper on Cell Death and Disease website (http://www.nature.com/cddis) 